Supporting information for

\title{
Atomistic modeling of PEDOT:PSS complexes II: Force field parameterization
}

\author{
Wesley Michaels, ${ }^{* \dagger}$ Yan Zhao, ${ }^{\ddagger}$ and and Jian Qin, ${ }^{*, \dagger}$ \\ $\dagger$ Department of Chemical Engineering, Stanford University, Stanford, CA 94305 \\ $\ddagger$ State Key Laboratory of Silicate Materials for Architectures, Wuhan University of \\ Technology, Wuhan 430070, China
}

E-mail: wpm216@stanford.edu; jianq@stanford.edu

\section{Table of Contents}

Section S1: Objective function details for parameterization.

Section S2: Assessment of vibrational mode overlap for large molecules.

Section S3: Comparison of neutral and doped PEDOT vibrational spectra.

Section S4: Energy matching results for neutral $\mathrm{PEDOT}_{6}$.

Section S5: Effect of bonded and nonbonded parameters on flexibility of doped PEDOT. 


\section{Supporting Information}

\section{S1 Parameterization details}

The objective function being optimized is (reproduced from Equation 2 in the Main Text)

$$
f=w_{\mathrm{g}} f_{\mathrm{g}}+w_{\mathrm{v}} f_{\mathrm{v}}+w_{\mathrm{t}} f_{\mathrm{t}}+w_{\mathrm{e}} f_{\mathrm{e}}+w_{\mathrm{r}} f_{\mathrm{r}}
$$

Optimization weights $w_{\mathrm{g}}, w_{\mathrm{v}}, w_{\mathrm{t}}$, and $w_{\mathrm{e}}$ are as defined in the main text, corresponding to ground-state geometry, vibrational spectrum, torsional spectra, and energy-matching training data. Regularization is weighted by $w_{\mathrm{r}}$. The objective function components $f_{i}$ are defined as

$$
\begin{aligned}
& f_{\mathrm{g}}=\sum_{i \in\{\mathrm{B}, \mathrm{A}, \mathrm{D}, \mathrm{I}\}} c_{\mathrm{g}, i}\left(\frac{\sum_{j}^{N_{i}}\left(x_{i j}^{\mathrm{ref}}-x_{i j}\right)^{2}}{N_{i}}\right)^{1 / 2} \\
& f_{\mathrm{v}}=\sum_{i=1}^{234} c_{\mathrm{v}, i}\left(\tilde{\nu}^{\mathrm{ref}}-\tilde{\nu}_{i}\right)^{2} \\
& f_{\mathrm{t}}=\sum_{i \in N_{\mathrm{t}}} c_{\mathrm{t}, i}\left(\left(E_{i}^{\mathrm{ref}}-E_{i, \mathrm{~min}}^{\mathrm{ref}}\right)-\left(E_{i}-E_{i, \mathrm{~min}}\right)\right)^{2} \\
& f_{\mathrm{e}}=\frac{\sum_{i \in N_{\mathrm{s}}}\left(E_{i}-E_{i}^{\mathrm{ref}}\right)^{2}}{\sum_{i \in N_{\mathrm{s}}}\left(E_{i}^{\mathrm{ref}}-\bar{E}_{i}^{\mathrm{ref}}\right)^{2}} \\
& f_{\mathrm{r}}=\sum_{i \in\{\mathrm{B}, \mathrm{A}, \mathrm{D}, \mathrm{I}\}} \sum_{j}\left(\frac{p_{i, j}^{0}-p_{i, i}}{c_{r, j}}\right)^{2} .
\end{aligned}
$$

Parameters are defined as follows. A sum defined by $i \in\{\mathrm{B}, \mathrm{A}, \mathrm{D}, \mathrm{I}\}$ is a sum over all sets of bond, angle, dihedral, and improper dihedral interactions in the molecule. Geometric values of such interactions in Equation (S1) (i.e. the value of the parameter as measured in the ground-state geometry), are denoted by $x$. Variables superscripted with "ref" indicate that the value is from a reference calculation, whereas variables without a superscript are model

predictions. The number of each type of geometric value is denoted by $N_{i}$ (this is e.g. the 
number of bonds in the molecule). In contrast, "equilibrium" values in Equation (S5), (i.e. the equilibrium constants in the equation for the energies of each bonded interaction), are denoted by $p$. The number of each type of equilibrium value is denoted by $n_{i}$ (this is e.g. the number of unique bond types in the force field).

Equation (S1) measures the root mean square displacement between predicted and equilibrium geometries, measured by differences in internal coordinates. The geometric weights $c_{\mathrm{g}, i}$ are 100 for bonds, 1/3 for angles, 0.1 for dihedrals, 0.1 for improper dihedrals. In Equation (S5), the L2 regularization weights $c_{\mathrm{g}, i}$ are $100 \mathrm{~kJ} /\left(\mathrm{mol} \cdot \mathrm{nm}^{2}\right)$ for bond force constants, $0.1 \mathrm{~nm}$ for bond equilibrium constants, $10 \mathrm{~kJ} /\left(\mathrm{mol}^{\left.- \text {degree }^{2}\right)}\right.$ for angle force constants, 2 degrees for angle equilibrium constants, $1 \mathrm{~kJ} / \mathrm{mol}$ for dihedral force constants, and $1 \mathrm{~kJ} / \mathrm{mol}$ for improper dihedral force constants. The superscript " 0 " indicates an initial value for a parameter, which is taken from GAFF.

Equation (S2) computes the total squared error in vibrational energies. The sum is performed across all 234 modes of the PEDOT hexamer $(d=3 \cdot 80-6=234)$, each with an energy $\tilde{\nu}_{i}$. Each mode is assigned a weight $c_{\mathrm{v}, i}$, which is determined by the function $c_{\mathrm{v}, i}=\left(1-\exp \left(-\tilde{\nu}_{i}^{\text {ref }} / k_{\mathrm{B}} T\right)\right)^{-1}$, where $k_{\mathrm{B}}$ is Boltzmann's constant and $T$ is a temperature. We set $T=300 \mathrm{~K}$ for the $\mathrm{PEDOT}_{6}{ }^{2+}$ optimization and $T=2000 \mathrm{~K}$ for the $\mathrm{PEDOT}_{6}$ optimization. This structure weights low-energy modes more heavily than high-energy ones (the disparity in weights increases with temperature), which was necessary to maintain an acceptable quality of fit in low-energy modes.

Equation (S3) computes the total squared error of all possible torsional scans of the molecule (of which there are $N_{\mathrm{t}}$ ) between the model and reference calculations. For a given torsion scan, each energy model and reference energy is taken relative to the minimum energy in that scan, indicated with the subscript "min." All torsions are assigned a weight $c_{\mathrm{t}, i}=1$ with the exception of inter-monomer backbone torsions, which are given $c_{\mathrm{t}, i}=10$.

Equation (S4) computes the total squared error of the difference in single point energies between the model and the reference for a series of snapshots drawn from a simulation (of 
which there are $N_{\mathrm{s}}$ ). The error is weighted by the variance of energies in the reference data; $\bar{E}^{\text {ref }}$ denotes the average energy of the reference data.

\section{S2 Vibrational mode overlap}

Our reported fitting of vibrational spectra entails minimizing the error between the sets of vibrational energies produced by MM and DFT. The prediction of dipole-transition coupling, and therefore of transition intensity, is unreliable in a classical nonpolarizable force field. Furthermore, overlap of vibrational modes, defined as the inner product of the normal displacements for a given mode predicted by DFT and by MM, is low for both GAFF and the optimized force fields. This result is expected due to the high dimensionality of the vibrational modes. We did not include normal mode overlap nor transition intensities as components of the objective function for these reasons.

We explain here why the overlap between high-dimensional vibrational vectors can be expected to be low. We examine a normal displacement vector,

$$
\mathbf{a}_{i}=\left(\delta_{x 1}, \delta_{y 1}, \delta_{z 1}, \delta_{x 2}, \delta_{y 2}, \delta_{z 2}, \ldots, \delta_{x 80}, \delta_{y 80}, \delta_{z 80}\right)_{i}
$$

defined by the set of Cartesian displacements for each atom in the 80-atom PEDOT hexamer. Each $\mathbf{a}_{i}$ is related to an eigenvector of the the molecular Hessian, $\mathbf{H}_{i j}$. For our purposes, all vectors $\mathbf{a}_{i}$ come from a DFT calculation. Including translational and rotational modes, the hexamer has $3 \cdot 80=240$ of such modes (which we label $\mathbf{a}_{i}$, for $1 \leq i \leq 240$ ) that form an orthonormal set. We define $\mathbf{a}_{i}^{\prime}$ as an approximation to $\mathbf{a}_{i}$, which represents the prediction of an MD model. Here, we assume there is no systematic error in the model prediction, and that we may approximate each element of $\mathbf{a}_{i}^{\prime}$ as $a_{i j}^{\prime}=a_{i j}+\varepsilon_{i j}$, where $\varepsilon_{j}$ is sample from a random distribution, e.g. the normal distribution. This representation is approximate because it does not guarantee that the set of all $\mathbf{a}_{i}^{\prime}$ vectors is orthogonal. 
The magnitude of each $\mathbf{a}^{\prime}$ is

$$
\left|\mathbf{a}_{i}^{\prime}\right|=\sum_{j}^{d}\left(a_{i j}+\varepsilon_{i j}\right)\left(a_{i j}+\varepsilon_{i j}\right),
$$

where $d$ is the dimensionality of the vector $\left(d=240\right.$ for $\left.\mathrm{PEDOT}_{6}\right)$. For a symmetricallydistributed $\varepsilon$ and sufficiently large $d$, we can normalize $\mathbf{a}^{\prime}$ by a factor

$$
C=\sum_{j}^{d}\left(a_{i j}^{2}+\varepsilon_{i j}^{2}\right) .
$$

The expected alignment of $\mathbf{a}$ and $\mathbf{a}^{\prime}$ is therefore

$$
\left\langle\mathbf{a}_{\mathbf{i}} \cdot \mathbf{a}_{i}^{\prime}\right\rangle=\left\langle\sum_{j}^{d} a_{i j} \frac{a_{i j}+\varepsilon_{i j}}{\sum_{k}^{d}\left(a_{i k}^{2}+\varepsilon_{i k}^{2}\right)}\right\rangle=\left\langle\frac{\sum_{j}^{d} a_{i j}^{2}}{\sum_{k}^{d}\left(a_{i k}^{2}+\varepsilon_{i k}^{2}\right)}\right\rangle=\frac{1}{1+d\left\langle\varepsilon_{j}^{2}\right\rangle},
$$

where we have used the (assumed) symmetry and independence of $\varepsilon$, as well as the orthonormality of a, to make the simplifications. Thus, as $d$ increases, the expected alignment monotonically decreases. Systematic inaccuracies in the model arising from, for example, the overestimation of bond strengths, will only degrade the quality of fit. We expect that as the dimensionality of the normal modes increases, there are greater opportunities for these to arise, as well. 


\section{S3 Neutral and doped PEDOT Vibrational Spectra}
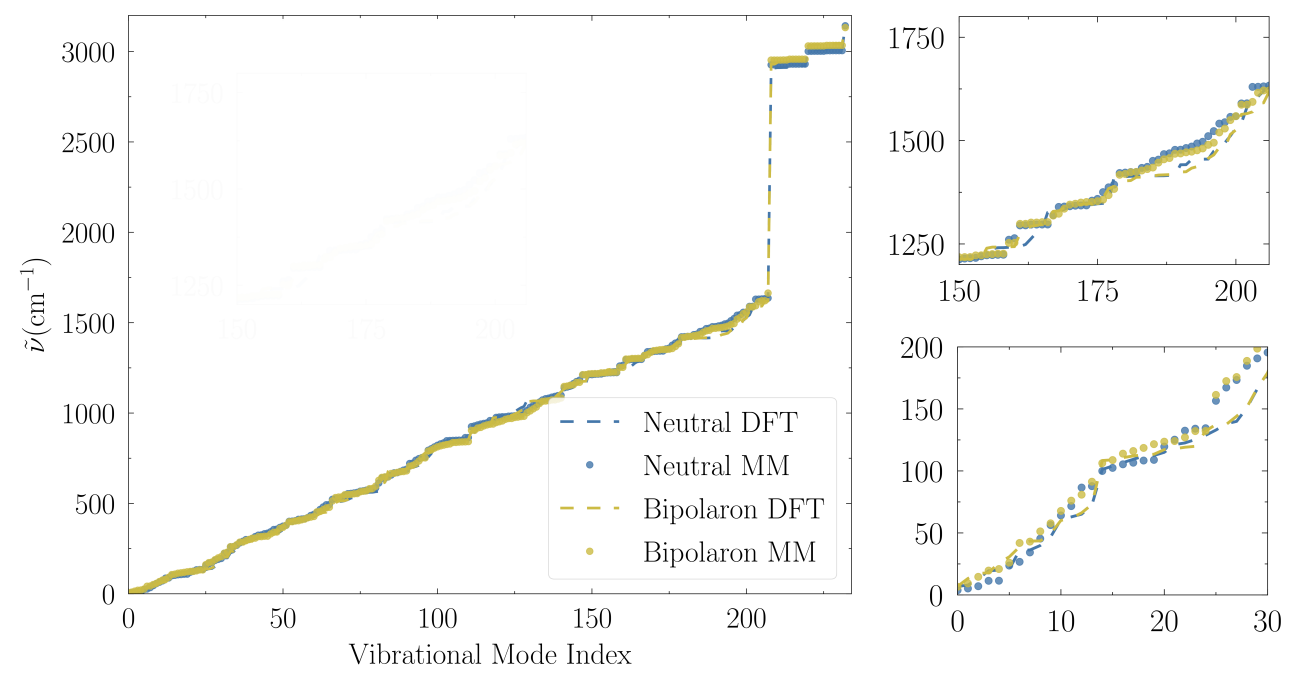

Figure S1: Vibrational spectra of $\mathrm{PEDOT}_{6}$ (blue) and $\mathrm{PEDOT}_{6}{ }^{2+}$ (yellow) calculated by their optimized force fields (points) and $\omega \mathrm{B} 97 \mathrm{x}-\mathrm{D}$ (dashed line). Insets: subset of $\mathrm{PEDOT}_{6}{ }^{2+}$ vibrational spectrum, focusing on (top) low-energy vibrational modes and (bottom) modes related to perturbations of CP backbone.

Despite differences in other observables, such as molecular charge and torsional barriers, the vibrational energies of $\mathrm{PEDOT}_{6}$ and $\mathrm{PEDOT}_{6}{ }^{2+}$ in Figure $\mathrm{S} 1$ appear quite similar across the entire range of modes for both molecules. This observation can be rationalized by considering that the chemical structure of the molecule has not changed, and, as a result, that the same set of chemical bonds is being perturbed when the molecule vibrates. Though we demonstrate in the main text that in a harmonic approximation the force constants of each bond differ between $\mathrm{PEDOT}_{6}$ and $\mathrm{PEDOT}_{6}{ }^{2+}$, the number of "double bonds" in PEDOT remains constant after a shift from an aromatic $\left(\mathrm{PEDOT}_{6}\right)$ to quinoid $\left(\mathrm{PEDOT}_{6}{ }^{2+}\right)$ configuration. Because Figure S1 shows the vibrational spectra in order of increasing vibrational energy, it is not surprising that the range of energies in $\mathrm{PEDOT}_{6}$ and $\mathrm{PEDOT}_{6}{ }^{2+}$ is similar.

Such an understanding further suggests that the normal modes themselves would change between $\mathrm{PEDOT}_{6}$ and $\mathrm{PEDOT}_{6}{ }^{2+}$, however. That is indeed the case, as evidenced by two points. First, we consider the alignment of normal modes of $\mathrm{PEDOT}_{6}$ and $\mathrm{PEDOT}_{6}{ }^{2+}$, 
defined by

$$
x_{i}=\left|a_{i}^{n} \cdot a_{i}^{b}\right|
$$

The vectors $a_{i}^{n}$ and $a_{i}^{b}$ represent the $i^{\text {th }}$ normal mode of the $\mathrm{PEDOT}_{6}$ and $\mathrm{PEDOT}_{6}{ }^{2+}$ vibrational spectra (neglecting rotational and translational modes), as defined in Eq. (S6). The average $\bar{x}$ can be taken across all 234 modes of each molecule. For the data as presented in Figure $\mathrm{S} 1, \bar{x}=0.37$. Because the sets of eigenvectors $\left\{a^{n}\right\}=\mathbf{A}^{n}$ and $\left\{a^{b}\right\}=\mathbf{A}^{b}$ are orthonormal matrices (including rotational and translational modes), a value of $\bar{x}=0$ represents no overlap, whereas a value of $\bar{x}=1$ indicates perfect overlap. Figure S2(a), showing the overlap of each mode, demonstrates that no systematic trend exists to predict which modes strongly or weakly overlap. Rearranging the modes to maximize overlap using the Hungarian Algorithm increases $\bar{x}$ to 0.50. Though this is a material increase, it nonetheless indicates that overlap is incomplete.

Second, recognizing that both $\mathbf{A}^{n}$ and $\mathbf{A}^{b}$ (including rotational and translational modes) span the same vector space, we must consider whether the energy of a given mode of $\mathrm{PEDOT}_{6}$ can be predicted by its projection onto the $\mathbf{A}^{b}$ basis. To do so, we estimate the vibrational energy of each $a_{i}^{n}$, denoted as $\hat{\tilde{\nu}}_{a_{i}}^{n}$, by $\hat{\tilde{\nu}}_{a_{i}}^{n}=\left(\left|\mathbf{A}^{b+} \mathbf{a}_{i}^{n}\right|\right) \cdot \Theta_{b}$, where $\mathbf{A}^{b+}$ is the Moore-Penrose inverse of $\mathbf{A}^{b}, \Theta^{b}$ is the vector of $\mathrm{PEDOT}_{6}{ }^{2+}$ vibrational energies shown in Figure S1, and $|\cdots|$ denotes an absolute value. Figure S2(c) clearly shows that $\hat{\tilde{\nu}}_{a_{i}}^{n}$ is not a good estimator for $\tilde{\nu}_{a_{i}}^{n}$, underscoring that the vibrational spectra of $\mathrm{PEDOT}_{6}$ and $\mathrm{PEDOT}_{6}{ }^{2+}$ are indeed different, despite the similarity of their representations in Figure S1. 
(a)

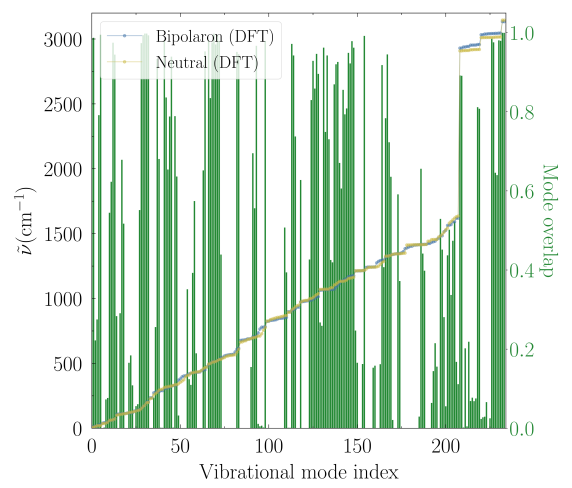

(b)

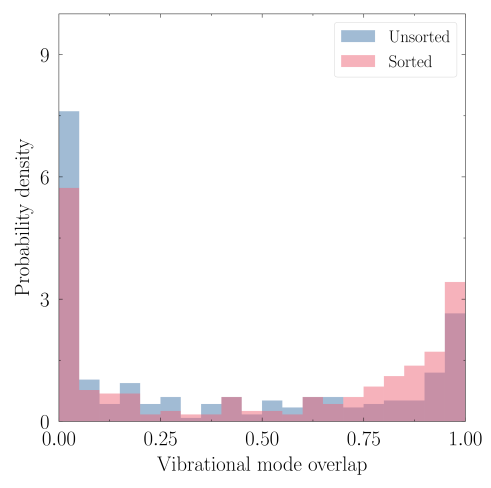

(c)

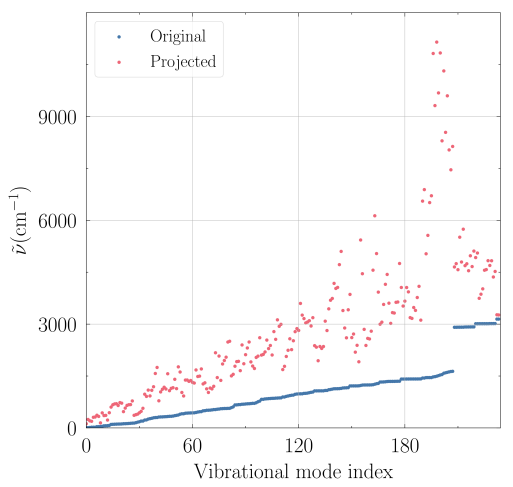

Figure S2: Analysis of differences in vibrational spectra between $\mathrm{PEDOT}_{6}$ and $\mathrm{PEDOT}_{6}{ }^{2+}$. (a) Direct comparison of spectra. Bipolaron and neutral spectra are shown in blue and yellow, respectively, with energies displaced on the primary y-axis. The overlap in each vibrational mode (Eq. (S7)) is shown as a single green bar, whose height is measured on the secondary y-axis. (b) Histogram of overlap values in (blue) unaltered spectra and (red) spectra after sorting with the Hungarian Algorithm to maximize overlap. (c) Comparison of the energies of true (blue) and projected (red) neutral vibrational spectra. See text for a procedural description. 


\section{S4 Neutral PEDOT energy matching}

(a)

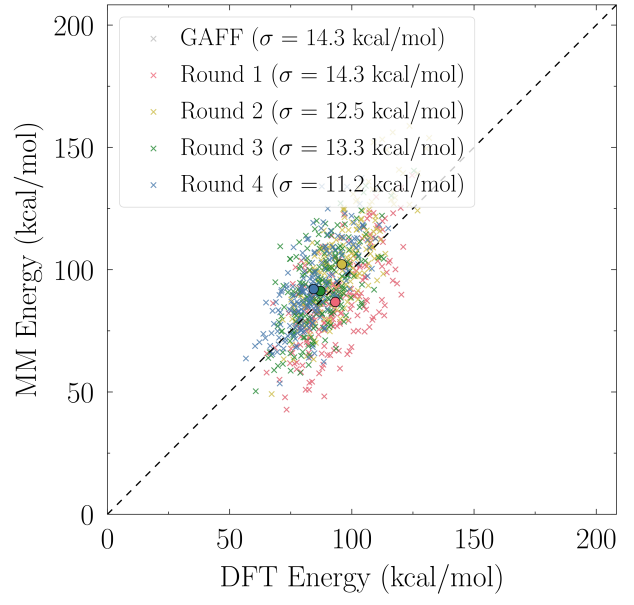

(b)

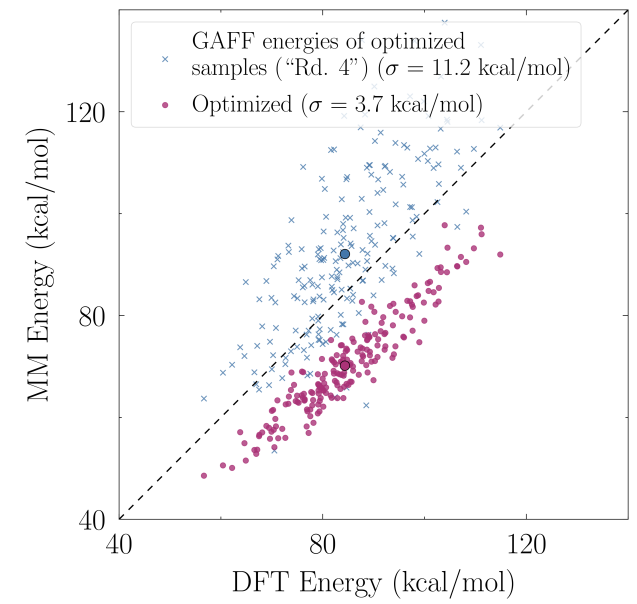

Figure S3: Energy matching results for $\mathrm{PEDOT}_{6}$. (a) Improvement of the PES with successive iterations. ("Rd." is an abbreviation for "round".) Shown are GAFF energies of configurations sampled with the optimized force field after each iteration. All DFT data are referenced to the ground state energy, calculated at the DF-DSD-PBEP86/juncc-pV $(\mathrm{T}+\mathrm{d}) \mathrm{Z} / / \mathrm{PBE} 0 /$ jun-cc-pVDZ level of theory. (b) Performance of GAFF vs. the optimized $\mathrm{FF}$ on the final test data set. Bolded points indicate the centroid of each distribution. Standard deviations $(\sigma)$ are of the residuals from the unity line.

Figure S3 shows the results of successive optimization rounds (Fig. 8(a)). Fig. S3(a) shows that though GAFF samples are centered close to the unity line, their energies have high variance. Optimization (Fig. S3(b)) significantly improves this issue, yielding a standard deviation of the residuals to test data of $3.7 \mathrm{kcal} / \mathrm{mol}$, compared to the $11.2 \mathrm{kcal} / \mathrm{mol}$ of GAFF. 


\section{S5 Doped PEDOT conformational flexibility}

(a)

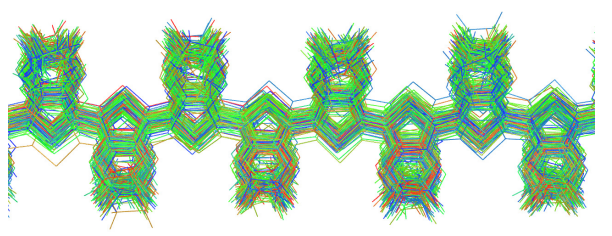

(c)

\section{(c)}

(b)

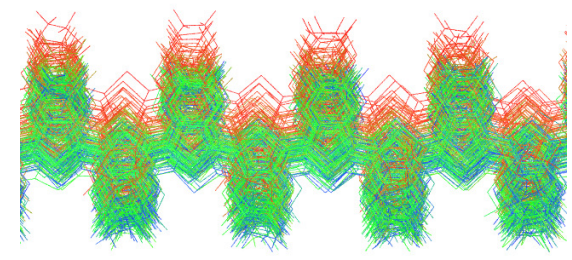

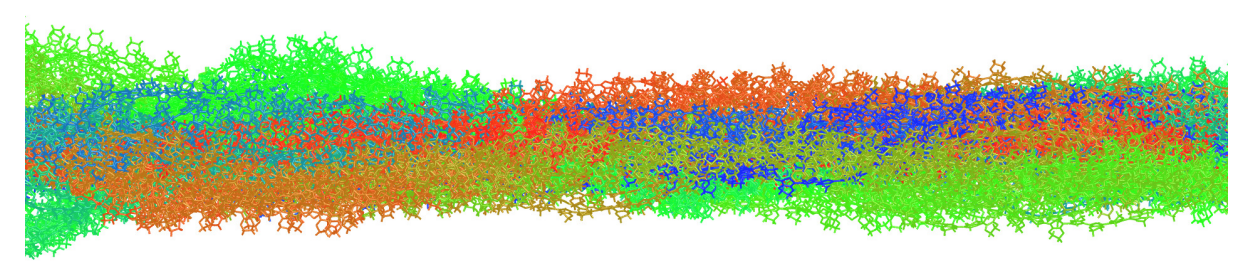

Figure S4: Configurations of PEDOT in various environments. Panels (a-c) show representative segments of the backbones of (a) highly-doped PEDOT $_{120}$ using the force field optimized in this work; (b) highly-doped PEDOT 120 using the force field optimized in this work, with all partial charges set to zero; (c) neutral PEDOT 120 using the force field optimized in this work.

Figure 54 shows representative segments of $\mathrm{PEDOT}_{120}$ backbones simulated with different parameter sets. It is evident that the highly-doped polymer with zero partial charges (Fig. S4(b)) adopts behavior more similar to the highly-doped polymer with partial charges (Fig. S4(a)) than to the undoped polymer (Fig. S4(c)). Because the highly-doped polymers have identical bonded terms (differing only in partial charge values), this comparison highlights the influence of the bonded parameters, which arise directly from the doping effects in the training data, on the overall chain structure. 